David S. Landes, L'heure qu'il est. Les horloges, la mesure du temps et la formation du monde moderne Paris, Les Belles Lettres, 2017Université Clermont-Auvergne

\title{
Catherine Cardinal
}

\section{(2) OpenEdition Journals}

Édition électronique

URL : http://journals.openedition.org/artefact/1730

DOI : $10.4000 /$ artefact. 1730

ISSN : 2606-9245

Éditeur :

Association Artefact. Techniques histoire et sciences humaines, Presses universitaires du Midi

\section{Édition imprimée}

Date de publication : 30 mai 2018

Pagination : 294-297

ISBN : 978-2-7535-7494-6

ISSN : 2273-0753

\section{Référence électronique}

Catherine Cardinal, «David S. Landes, L'heure qu'il est. Les horloges, la mesure du temps et la formation du monde moderne », Artefact [En ligne], 7 | 2017, mis en ligne le 14 février 2019, consulté le 23 septembre 2020. URL : http://journals.openedition.org/artefact/1730 ; DOI : https://doi.org/10.4000/ artefact. 1730

Ce document a été généré automatiquement le 23 septembre 2020.

\section{cc) (†) $\ominus$}

Artefact, Techniques, histoire et sciences humaines est mise à disposition selon les termes de la Licence Creative Commons Attribution - Pas d'Utilisation Commerciale - Pas de Modification 4.0 International. 


\title{
David S. Landes, L'heure qu'il est. Les horloges, la mesure du temps et la formation du monde moderne
}

\author{
Paris, Les Belles Lettres, 2017Université Clermont-Auvergne
}

\section{Catherine Cardinal}

\section{RÉFÉRENCE}

David S. Landes, L'heure qu'il est. Les horloges, la mesure du temps et la formation du monde moderne, Paris, Les Belles Lettres, 2017, 637 p.

1 En 1983, David S. Landes (1924-2013) publia Revolution in Time, Clocks and the Making of the Modern World. Quatre années après, il en offrit une version française, revue et augmentée, L'heure qu'il est. Les horloges, la mesure du temps et la formation du monde moderne. Cette version est à nouveau proposée par les Belles Lettres avec un cahier central de 104 illustrations, la plupart en couleurs alors qu'elles étaient en noir et blanc dans la première édition.

2 Professeur d'histoire et d'économie à l'université d'Harvard jusqu'en 1996, Landes se spécialisa dans l'histoire économique et technique de l'Europe comme en témoigne son ouvrage, L'Europe technicienne ou le Prométhée libéré. Sa passion des montres s'affirma vers 1970. Comme il le dit dans sa préface de 1983, «Venu d'une civilisation utilitariste, j'étais ébahi à la pensée du talent et du temps qu'il avait sûrement fallu pour fabriquer ces objets, de ce soin, de ce fini... ». Il collectionna et, simultanément, il s'intéressa à l'histoire de l'horlogerie. En 1978, après un séminaire à l'université de Zurich sur le sujet, il eut l'idée de l'ouvrage, Revolution in Time. Il s'agit d'une histoire de la mesure du temps $\mathrm{du} \mathrm{x}^{\mathrm{e}}$ au $\mathrm{xx}^{\mathrm{e}}$ siècle, principalement vue dans son contexte social, technique et économique. Comme l'annonce Landes, "C'est un triptyque: une étude d'histoire culturelle (pourquoi l'horloge mécanique a-telle été inventée en Europe ?) ; d'histoire de la science et de la technique (comment est-on passé de garde-temps grossiers et 
approximatifs à des instruments de haute précision?) ; et d'histoire économique et sociale (qui a fait ces instruments ? comment? qui s'en est servi ? pourquoi ?)» (p. 19).

Dans l'introduction, des constats se succèdent sur l'importance de la mesure du temps dans la vie ordinaire, les techniques et les sciences. D'emblée, l'horloge mécanique, apparue au XIII ${ }^{\mathrm{e}}$ siècle, est reconnue par l'auteur comme « une des grandes inventions de l'histoire de l'humanité » (p. 34).

4 La première partie, « Trouver le temps ", démontre pourquoi l'horloge mécanique a été inventée en Europe et non en Chine. Landes revient sur l'invention sans lendemain de l'horloge astronomique, à roue hydraulique, de Su Song à la fin du XI siècle et réfute les arguments de Joseph Needham visant à considérer de telles horloges comme les prémices de l'horlogerie mécanique. Une bonne fois pour toutes, il faut considérer que l'horloge à poids-moteur et échappement à foliot marque les débuts d'une technologie nouvelle. Le second chapitre immerge encore le lecteur dans l'empire chinois ; Landes analyse la réception, tour à tour empreinte d'admiration et de mépris, des horloges occidentales depuis l'époque où le père Ricci les offrait pour faciliter la diffusion du christianisme. Malicieusement intitulé "Frère Jacques dormez-vous», le chapitre suivant aborde l'apparition des horloges mécaniques, reflet du progrès de la mesure du temps en réponse aux règles de la vie monastique ainsi qu'aux rythmes de travail dans les villes. Les cloches qui proliféraient furent avantageusement remplacées par les horloges de tour sonnant des heures égales. «Les heures égales annonçaient la victoire d'un nouvel ordre politique, culturel et économique » (p. 125).

La deuxième partie, "Garder le Temps ", montre comment un appareil volumineux, inexact, de facture grossière s'est métamorphosé en un instrument portatif, précis, régulier. La miniaturisation de l'horloge permise par l'utilisation d'un ressort-moteur est une première étape, située vers 1430. Le processus s'accentua pour donner naissance à la montre dans le dernier quart du XvI siècle. Landes souligne l'importance de la miniaturisation, « aiguillon technique » qui nécessite des qualités exceptionnelles d'adresse. Il remarque les conséquences sociales et culturelles de l'usage des montres qui engendre une privatisation du temps. L'auteur s'arrête sur les horloges à automates fabriquées sous la Renaissance, à Augsbourg et sur les montres-bijoux créées à Blois, Paris, Genève. Ces «jouets, ornements" ne doivent pas faire oublier le principe directeur de l'évolution de l'horlogerie: le perfectionnement du mouvement. L'exigence de précision émanait non seulement des "sociétés mercantiles et bourgeoises " mais aussi des astronomes et des navigateurs. Les inventions de Huyghens, le pendule (1656) et le spiral-réglant (1675) sont longuement détaillées. Après lui, les recherches se concentrèrent sur les problèmes liés aux changements de la température et à la friction des engrenages. Les travaux de Harrison, Graham, Ellicott, Fatio, Tompion sont autant de pas vers la conquête de l'exactitude. La meilleure expression de ce progrès se trouve dans la construction d'horloges suffisamment justes et régulières, quand elles sont embarquées sur un navire, pour servir au calcul de la longitude. Tout un chapitre est consacré aux horloges marines réalisées par John Harrison, Pierre Le Roy et Ferdinand Berthoud. Après eux, John Arnold et Thomas Earnshaw s'efforcèrent d'améliorer le chronomètre de marine, en le dotant d'un échappement à détente à ressort. Landes achève cette "histoire de la quête de la précision » sur la rivalité de ces deux horlogers qui eut des échos jusque dans une vente aux enchères de Christie's en 1980. 
6 Dans la dernière partie, la plus longue de son essai, David S. Landes reprend le fil de l'histoire depuis les horloges de clocher jusqu'aux montres à quartz en privilégiant les aspects économiques. Il s'interroge sur la construction et l'entretien des premières horloges de tour en prenant comme exemple celle de Perpignan. Se référant à l'horloge de Cambrai, il souligne que de tels ouvrages concouraient au prestige des villes. La division du travail retient son attention ; c'est d'abord une collaboration entre corps de métiers, horlogers, forgerons, charpentiers, orfèvres, menuisiers puis c'est une spécialisation à l'intérieur de chaque profession. "L'industrie horlogère se ramifia en se développant » (p. 282). L'Angleterre s'imposa à la fin du XVII ${ }^{e}$ siècle au détriment de la France, ce qui contraignit à l'émigration un grand nombre de ses horlogers. Landes met en valeur une pléiade d'horlogers, Thomas Tompion, Daniel Quare, George Graham, John Ellicott, Thomas Mudge, qui assurèrent la domination anglaise, sur le plan technique et commercial, entre 1680 et 1780. La France prit une certaine revanche avec des maîtres comme Julien et Pierre Le Roy, Ferdinand et Louis Berthoud, Jean-Antoine Lépine, Antide Janvier, Robert Robin et, surtout, Abraham-Louis Breguet. La concurrence la plus agressive vint de Genève qui organisa une fabrique englobant la ville et les régions avoisinantes. Genève elle-même subit la concurrence des centres de production jurassiens comme La Chaux-de-Fonds, Le Locle, Fleurier. Au XIX siècle, la Suisse affirma sa suprématie du fait de son opportunisme commercial conjugué au nombre croissant de ses horlogers de talent. Les horlogers suisses se remirent néanmoins en question face aux succès de l'horlogerie américaine fondés sur la fabrication mécanisée des montres. Un équilibre fut trouvé entre l'emploi des calibres ébauchés mécaniquement et la finition manuelle. Même si la Suisse perdit en partie le marché américain, sa domination se renforça quand les montres-bracelets se généralisèrent. «Les Suisses contrôlaient encore plus de la moitié du marché au début des années 1950 - une position qu'ils conservèrent avec des hauts et des bas, tout au long des années suivantes. » (p. 449).

7 «La Révolution du quartz», ainsi que Landes intitule son dernier chapitre, fut le nouveau défi que les Suisses eurent à relever. Les premières horloges à quartz des années 1930 étaient aussi volumineuses "qu'un bon réfrigérateur de restaurant " (p. 458); leur miniaturisation nécessita une trentaine d'années de recherches notamment menées par le Centre électronique horloger (Neuchâtel). De leur côté, les Américains et les Japonais adoptèrent rapidement la nouvelle technique. En quelques années, les Japonais (Seiko, Citizen) réussirent à diminuer le volume, abaisser le coût, réduire l'énergie du mouvement tout en incluant toutes sortes de complications (calendrier, réveil, chronographe, etc.). De 1970 à 1980, la production japonaise fut marquée par une forte croissance qui n'a d'égale que la chute des exportations suisses. En 1982, les entreprises d'horlogerie suisses étaient moins de 500 alors qu'elles étaient plus de 2000 en 1963. Il faut souligner la résistance des maisons de prestige qui perpétuaient la tradition des montres mécaniques de haute précision. Landes achève son récit par un événement qui stimula l'industrie suisse: la mise au point de la "Swatch» («Swiss Watch»), montre à quartz avec cadran à aiguilles et bracelet en plastique. Son mouvement et son boîtier étaient assemblés sur des chaînes automatisées. Son design en fit un produit de mode et même de collection.

Plutôt qu'une bibliographie, David S. Landes a opté pour des notes réunies sur une centaine de pages, en fin de volume, témoignant de la densité des informations réunies. Rappelons, en particulier, que Landes était un parfait francophone, au fait de toutes les 
publications parues en France et en Suisse et que sa personnalité avenante doublée d'une inlassable curiosité l'amena à fréquenter maints collectionneurs, marchands, conservateurs de musée. Sa manière de faire des constats qui "sautent aux yeux" avant d'entraîner le lecteur dans de subtils développements, son goût des mots simples et des formules percutantes sont caractéristiques de son écriture. La volonté de convaincre le lecteur et de soutenir son attention se traduit par l'emploi du je, des expressions familières et l'insertion d'anecdotes. Ce remarquable sens de la vulgarisation a, sans aucun doute, contribué au succès de l'ouvrage (le seul écrit dans ce domaine par Landes, un coup de maître).

L'ouvrage offre une synthèse de sept siècles de production horlogère en éclairant ses aspects sociaux et économiques, "une histoire négligée des spécialistes" (p.41). Landes tente ainsi de déceler les causes de la réussite de la Suisse, des États-Unis et du Japon dans l'industrie horlogère. Avec acuité, il observe la concurrence suisse au détriment de l'horlogerie anglaise, les menaces exercées par les entreprises américaines dans les années 1870 puis, cent ans plus tard, par les entreprises japonaises. En économiste averti, Landes décortique le développement de l'industrie horlogère dans ses lieux de production $d u x I_{I I}{ }^{e}$ au $x^{e}$ siècle. Il suppose que "la consommation d'horloges et de montres pourrait bien être le meilleur indice de la modernisation - meilleur encore que la consommation énergétique par tête... » (p. 434).

Trente ans ont passé depuis la parution. Il aurait été intéressant d'accompagner la réédition de quelques ajouts afin d'actualiser certains renseignements et de signaler des publications postérieures. Des réponses peuvent être apportées à quelques questions posées par Landes. Quand il se demandait si l'horloger George Daniels parviendrait à trouver la collaboration d'un industriel, on lui répond qu'il a travaillé avec Omega pour fabriquer ses montres à échappement co-axial (p. 469). Quand il posait la question, «Est-il possible de bâtir une nouvelle industrie horlogère sur les vestiges de l'ancienne?", il suffit d'observer, depuis les années 1990, la vitalité des maisons suisses, héritières des prouesses techniques des XVIII et XIX ${ }^{e}$ siècles, affichée au Salon mondial de l'horlogerie, à Bâle, et au Salon international de la Haute Horlogerie, à Genève. L'horlogerie mécanique, érigée au niveau d'un art, telle qu'elle apparait annuellement au Grand Prix de Genève, offre aux historiens un nouveau chapitre à écrire, aussi exaltant que les précédents. 